\title{
Uso de la herramienta «CMAPS TOOLS» como ayuda para la comprensión lectora de alumnado de Primaria
}

\author{
Use of the "CMAPS TOOLS» tool as a help for the reading comprehension of \\ Elementary School students
}

\author{
Esther María González Castellón \\ e-mail: esther.gonzalez@unir.net \\ Universidad Internacional de La Rioja. España
}

Noelia Carbonell Bernal

e-mail: noelia.carbonell@unir.net Universidad Internacional de La Rioja. España
María Cinta Aguaded Gómez (iD)
e-mail: maguaded@nebrija.es
Universidad Nebrija. España

\author{
Gemma Teresa Asensio Quirant \\ e-mail: gemma.quirant@uchceu.es
}

Universidad CEU Cardenal Herrera. España

\begin{abstract}
Resumen
Los alumnos de primaria con dificultades específicas de aprendizaje relacionadas con la lectoescritura necesitan que se les facilite una respuesta educativa adaptada que les permita alcanzar el éxito escolar. Los datos de prevalencia de la dislexia giran en torno al 5-10\%, llegando incluso al 15\%, esto quiere decir que en una clase de Educación Primaria cuanta al menos un alumno con esta dificultad. Los docentes no encuentran estrategias efectivas para trabajar con ellos dentro del grupo clase, así, el objetivo principal de la presente investigación es comprobar el aporte que realiza la herramienta de Cmaps Tools tanto en el alumnado con dislexia como para el resto del grupo clase. La muestra consta de un total de 47 estudiantes de $2^{\circ}$ y $3^{\circ}$ de educación primaria, entre los cuales se encuentran 7 alumnos con dislexia. Tras realizar el estudio, se constata como la utilización de TIC y mapas conceptuales mejoran tanto la comprensión lectora de los alumnos que presentan dislexia como en la totalidad del grupo, desarrollándose a la vez aspectos como son las habilidades socioafectivas y un aumento en los niveles de motivación y autoestima en el alumnado.

Palabras clave: dislexia; comprensión lectora; TIC; cmaps tools; dificultades especificas de aprendizaje.
\end{abstract}

\section{Abstract}

Dyslexic students have specific learning difficulties related to literacy and need to be provided with a different educational response that allows them to achieve school success. Dyslexia prevalence data revolves around 5$10 \%$, even reaching 15\%. This means that, in a Primary Education class, it is easy to find at least one student with dyslexic difficulties. The main objective of this research is to verify the contribution made by the Cmaps Tools tool, both in students with dyslexia and in classmates who do not have learning difficulties. The sample consists of a total of 47 second and third grade school students, among whom 7 of those students suffer dyslexia. After completing the study, we see how the use of ICTs and concept maps improve both the reading comprehension of the students who present dyslexia, as well as how important aspects such as socio-affective skills are developed, and with this, an increase of motivation and self-esteem of the students is achieved. These aspects must be of vital importance, in order for the student to obtain a good emotional development. Keywords: dyslexia; reading comprehension; ICT; cmpas tools; specific learning difficulties.

Recibido / Received: 08-07-2020

Aceptado / Accepted: 19-11-2020

Publicado en línea / Published online: 22-12-2020

Cómo referenciar este artículo / How to reference this article:

González, E. M., Carbonell, N., Aguaded, M. C., \& Asensio, G. T. (2021). Uso de la herramienta «CMAPS TOOLS» como ayuda para la comprensión lectora de alumnado de Primaria. Tendencias Pedagógicas, 37, pp. 145-155. doi: 10.15366/tp2021.37.012 


\section{Introducción}

Un sistema educativo inclusivo se identifica por ser capaz de dar una respuesta educativa de calidad a todo el alumnado, independientemente de las circunstancias personales o sociales. Dentro de los alumnos escolarizados en los centros educativos, nos encontramos con el alumnado con necesidades específicas de apoyo educativo, entendido éste como aquél que, según recoge la Ley orgánica 2/2006, de 3 de mayo, de Educación, en su texto modificado por la Ley Orgánica 8/2013, de 9 de diciembre, para la mejora de la calidad educativa, en su artículo 71.2, que durante su escolarización, requiera de una atención educativa diferente a la ordinaria, por presentar necesidades educativas especiales, por dificultades específicas de aprendizaje, TDAH, por sus altas capacidades intelectuales, por haberse incorporado tarde al sistema educativo, o por condiciones personales o de historia escolar.

La problemática que implica la dislexia a la hora de superar algunos de los aspectos dentro de los contenidos educativos, ha hecho que durante estos últimos años esta dificultad, sea un tema de estudio, tanto por la dificultad en la lectoescritura al igual que por el gran número de niños a los que les afecta (Scrich et al., 2017).

Dar una adecuada respuesta educativa a este alumnado implica, primero, el conocimiento de sus características, sus necesidades y las estrategias más eficaces para una adecuada atención educativa. Por ello, los docentes necesitan ampliar y mejorar la formación que reciben en el ámbito de las dificultades de aprendizaje para llegar a ser eficaces a la hora de prevenir y solucionar los obstáculos que el alumnado deba superar durante dicho proceso (González, 2009).

La correcta utilización de las TIC y mapas conceptuales, favorecen el aprendizaje en el alumnado, dado que estas herramientas con un uso correcto y específico, crea en los discentes un aumento de la motivación, así como un instrumento facilitador de los contenidos, siendo a su vez, atractivo para los sujetos. No es suficiente que este tipo de alumnado reciba apoyo por especialistas fuera del aula, sino que dentro del grupo clase el tutor sea capaz de suministrarle los recursos necesarios para poder ir superando sus dificultades realizando actividades que puedan beneficiar también al resto de alumnos.

En un aula se debe tener un alto clima motivacional, esta cuestión hace que el alumnado mejore tanto en su nivel académico como emocional y para ello, el profesorado no puede centrarse únicamente en la transmisión del contenido, de los conocimientos, debe ir más allá, debe emplear técnicas de enseñanza, así como ser empáticos y motivadores con sus discentes (Pascual, 2016).

Según Bisquerra (2003), la motivación hay que encontrarla a partir de la emoción, esto implica tener desarrollada la Inteligencia Emocional, ya que esta crea un gran papel importante en el crecimiento de las personas y para poder conseguir estas habilidades y conocimiento, es necesario del empleo de cinco habilidades básicas tales como son el autoconocimiento, el autocontrol, la automotivación, la empatía y las habilidades sociales (Carbonell \& Cerezo, 2019).

La dificultad de la lectoescritura genera falta de motivación en el individuo, asimismo problemas de autoestima e incluso bajo rendimiento académico, siendo esto un grave problema no únicamente porque se ven gravemente afectados sus niveles académicos sino pueda afectar en niveles emocionales.

Uno de los problemas que se puede presentar en el alumno disléxico, es llegar a sufrir niveles de ansiedad, miedo o estrés, ya que estas emociones negativas surgen frente a una situación complicada en el aula (Quirant, Pascual \& Carbonell, 2020). El miedo a ser juzgado puede conducir a estos estados, como consecuencia a futuras reacciones o evocar recuerdos anteriores por una mala experiencia. Se debe mencionar que la ansiedad es una respuesta puramente emocional la cual se manifiesta como forma de adaptación al medio.

No debemos dejar que este aspecto cree ansiedad en el alumnado, y menos que conduzca a una ansiedad mayor y más cuando hay herramientas que pueden ayudar a conseguir los objetivos que necesita el alumno. El problema de esta patología es que puede mostrarse en tres estados distintos, leve, moderada y severa (Quirant, Carbonell \& Pascual, 2020).

Evitar este tipo de situaciones ayudará al alumnado, tanto a nivel académico como emocional, puesto que la ansiedad es un estado o condición emocional desagradable (Reyes et al., 2017).

La autoestima, es un factor importante en el buen desarrollo del estudiante, poseer una baja autoestima, puede provocar en fracaso escolar. En el caso en concreto del alumnado con dislexia, este factor puede estar presente y más en ciertas edades. Algunas investigaciones indican que durante la 
adolescencia temprana suele presentar un nivel más bajo que el cual, suele aumentar en los próximos años de la persona (Reina, Oliva \& Parra, 2010).

Debemos hacer que el alumnado realice las actividades de una forma amena, atractiva, motivadora, pero a su vez debe de cumplir los objetivos que se pretende que se adquiera, en definitiva, modificar la metodología con el fin de que llegue esa transmisión del conocimiento que queremos aplicar de una forma que enriquezca diferentes aspectos, no únicamente los académicos, por tanto, es importante que a través de la motivación también sirva para estimular en valores sociales (Ulloa et al., 2018).

\section{Concepto de dislexia}

Se pueden encontrar muchas definiciones del concepto de dislexia y en todas ellas aparece una relación con las dificultades para aprender a leer y escribir en niños que presentan un coeficiente intelectual normal y sin la presencia de ningún déficit sensorial Thompson (1992, p. 45), define Dislexia como:

una grave dificultad con la forma escrita del lenguaje independiente de cualquier causa intelectual, cultural y emocional. Se caracteriza porque las adquisiciones del individuo en el ámbito de la lectura, la escritura y el deletreo, están muy por debajo del nivel esperado en función de su inteligencia y de su edad cronológica. Es un problema de índole cognitivo, que afecta a aquellas habilidades lingüísticas asociadas con la modalidad escrita, particularmente el paso de la codificación visual a la verbal, la memoria a corto plazo, la percepción de orden y la secuenciación.

La Organización Mundial de la Salud, a través de la CIE-10 (Clasificación Internacional de Enfermedades, 1992), incorpora la dislexia en el apartado de trastornos específicos del desarrollo de las habilidades escolares, adoptando el término para referirse a esta de trastorno específico de la lectura.

A partir de la publicación en 2013 del DSM-V, la dislexia pasa a tener un tratamiento más especializado al ser considerada como un trastorno "específico" de aprendizaje de la lectura. A partir de todas las definiciones explicadas anteriormente se puede concluir que: la dislexia se define como un problema en el proceso de decodificación, la cual se manifiesta por una lectura insegura y lenta, una dificultad en reconocer las palabras y una escasa comprensión, sin que exista aparentemente una dificultad tanto intelectual como emocional y cultural. Suele venir acompañado de otras dificultades como pueden ser en la lengua escrita (disgrafía), y que repercuten así en el aprendizaje de otras áreas.

\subsection{Tipos de dislexia}

Los tipos de dislexia son distintos dependiendo de los autores que las definen y las corrientes por las que se rigen. Actualmente muchos autores distinguen entre dos tipos: la dislexia adquirida y la evolutiva. La adquirida, es aquella que padecen las personas que tras haber aprendido a leer y a escribir pierden, en mayor o menor medida, algunas de las habilidades ya adquiridas debido a una lesión cerebral. Según Fawcett y Nicolson (2004), dentro de todos los trastornos el de la dislexia evolutiva es tanto el más extendido como investigado. La evolutiva, se da cuando el niño se encuentra en edad escolar, más concretamente cuando comienza el aprendizaje escolar considerando este tipo de dislexia como genética y congénita (Wolf, Vellutino \& Gleason, 2000b).

\section{Los mapas conceptuales como herramienta de mejora en la comprensión lectora}

Tras realizar la lectura, para el lector se hace necesario plantearse cuáles son las ideas principales del texto, cuáles las secundarias y qué relación se establece entre ellas; una vez resueltas estas dudas, el lector estará en disposición de decir que ha comprendido el texto (González \& Iraizoz, 2013). Los mapas conceptuales son una herramienta fundamental para organizar estas relaciones y dar respuesta a estas cuestiones porque generalmente los textos tienen una estructura lineal que suele dificultar a los lectores el establecer las relaciones oportunas dentro del texto, por ello la estructura bidimensional de los mapas conceptuales es capaz de jerarquizar dichas relaciones y facilitar la comprensión de la lectura. El uso de mapas conceptuales ayuda a los alumnos, acostumbrados a aprender de memoria o a hacerlo superficialmente, a convertirse en aprendices más profundos o con mayor significado; es decir, ayudan a que las personas aprendan cómo aprender. 
La utilización de esta herramienta como mejora en el aprendizaje, es un tema que ha despertado en diversos autores, así como la comprobación de este, así como la necesidad de la utilización de la tecnología (Cabañas \& Ojeda, 2002; Cañas \& Ford, 2014; Díaz, 2013; González, 2009; González \& Iraizoz, 2003; Lucas-Molina et al., 2017; Muñoz, Serrano \& Marín, 2014; Novak \& Gowin, 1988; Segovia, 2001; Segovia \& Rincón, 2011; Villalustre \& Del Moral, 2010). Por ello, se debe considerar un recurso pedagógico no únicamente para el alumnado que tiene dislexia.

\subsection{Recursos TIC para la comprensión lectora: los mapas mentales o mapas conceptuales}

Entre los recursos TIC para hacer mapas mentales, destacamos los siguientes: IMINDMAP, FREE MIND y WORDLE.

En primer lugar, citaremos a IMINDMAP, este software usa los métodos de mapeo mental de Tony Buzan para crear mapas agradables al cerebro para: organizar, pensamiento creativo y planificar. Está integrado con Microsoft Office permitiendo exportar sus mapas mentales hacia programas como el Word y Excel, crearlos como PDF, y páginas web. Otro programa de ordenador es el FREE MIND, el cual permite la elaboración de mapas mentales o de conceptos, el cual está programada en Java, y resulta muy útil en el análisis y recopilación de información o ideas generadas en grupos de trabajo, pues con él, es posible generar mapas mentales y publicarlos en internet como páginas HTML, java o insertarlos dentro de wikis. Y por último citaremos WORDLE. Este recurso online, tiene como función crear nubes de palabras, ideal para la Técnica Rodari de palabras inductoras.

\section{Objetivos de la investigación}

El objetivo general de este estudio sería mejorar la comprensión lectora y las habilidades socioafectivas del alumnado disléxico a través del uso de los mapas mentales, realizados estos a mano y /o mediante programas informáticos (Cmaps).

Para la consecución de este, establecemos los siguientes objetivos específicos:

- Averiguar cómo influyen las Tecnologías de la Información y la Comunicación en la educación y en la lectoescritura.

- Analizar la importancia del uso de mapas conceptuales con el alumnado disléxico.

- Proponer la introducción de cambios innovadores en la práctica docente para la mejora de los resultados y de los procesos educativos de estos alumnos.

- Comprobar si esta herramienta mejora aspectos de aprendizaje además de aspectos emocionales como la autoestima tanto en el alumnado de estudio como en el grupo

- Medir si esta herramienta es motivadora para el alumnado.

\section{Metodología}

Este estudio se enmarca en investigación acción, donde después de analizar la problemática del centro y detallar las necesidades surgidas del contexto del centro se implementan una serie de medidas innovadoras para provocar cambios necesarios.

Para ello se pasó un cuestionario al profesorado para recoger las necesidades surgidas, entre ellas, de manera notable, sobresalía la necesidad de formación y los recursos para la atención al alumnado con necesidades específicas de aprendizaje dentro del grupo.

Descrito el problema de investigación, se plantean los objetivos, la muestra y se configuran los talleres y los agentes implicados

Se realizó un diseño cuasi experimental de medidas repetidas pre-test y post-test. Para el análisis estadístico descriptivo de la muestra hemos utilizado los métodos descriptivos básicos, la muestra empleada es de 47 alumnos, siendo $48,5 \%$ chicos y 51,5\% chicas. Las fases seguidas utilizadas han sido la identificación del alumno, la utilización de CMap y, por último, la valoración de resultados post-test. En la investigación participan de lleno el profesorado del centro. 


\subsection{Participantes}

El presente estudio se ha llevado en un centro educativo público de la Comunidad Autónoma de la Región de Murcia para la etapa de Educación Primaria.

La muestra real de sujetos ha sido un total de 47 alumnos de edades comprendidas entre los 7 y 9 años, pertenecientes a los cursos de $2^{\circ}$ y $3^{\circ}$ de Educación Primaria. Se han organizado dos grupos: el número 1 para el alumnado de segundo de Primaria y el 2 para los de tercero de Primaria.

El grupo 1 se conforma de un total de 22 alumnos de los cuales 3 de ellos presenta dislexia frente al grupo 2, que se compone de un total de 25 alumnos y muestran dislexia 4 sujetos del total.

La muestra de profesorado que se utilizó para iniciar el estudio y conocer las necesidades del centro, la constituyo todo el profesorado de educación primaria, incluido equipo directivo, especialista en Pedagogía terapéutica, audición y lenguaje y coordinar TIC, un total de 23 sujetos, de los cuales 16 eran mujeres y 7 hombres.

\subsection{Temporalización}

La investigación comienza con la recogida de necesidades del centro mediante la aplicación de un cuestionario. Para ello se realiza una primera toma de contacto con la dirección del centro y se le explica el motivo del estudio.

Una vez obtenido los permisos se aplica el cuestionario a los sujetos seleccionado y se organiza la aplicación de los talleres, los horarios y los agentes implicad0os.

La aplicación de los talleres sobre la elaboración de mapas conceptuales y programas socioafectivos impartido al grupo clase (que constituye los sujetos de la muestra). Se desarrollará a lo largo de doce sesiones de una hora de duración aproximadamente cada una. El profesorado responsable de impartir los talleres del primer grupo serán los profesores de AL y PT, el encargado del segundo grupo será el coordinador TIC del centro.

Las fases que comprenden dicha aplicación son las siguientes:

- Primera fase: se desarrolla durante el primer trimestre. En esta fase tiene lugar la puesta en marcha de todos los protocolos de actuación para la identificación de alumnado con dislexia y las evaluaciones iniciales por parte de los profesores que imparten las áreas instrumentales, se implicaran el orientador del centro, los especialistas de PT (Pedagogía Terapéutica) y AL (Audición y Lenguaje). Durante esta primera fase también se realizarán cursos formativos para el profesorado sobre las dificultades específicas de aprendizaje y sobre el manejo de la herramienta informática Cmaps impartidos por los investigadores y el coordinador TIC del centro.

- Segunda fase: tendrá lugar en la primera mitad del segundo trimestre, desarrollándose dos programas de intervención: (a) Aplicación de talleres de elaboración de mapas conceptuales. (b) Un programa de integración socioafectiva, con el objetivo conocer, entender, adaptar y respetar al alumnado disléxico en su aula. El programa de integración socioafectiva ha sido un elemento muy beneficioso para todo el colectivo, trabajándose la autoestima, el autoconcepto y habilidades sociales... no se ha utilizado solo con los alumnos de dislexia sino con todos los compañeros, y se trabajaban como una tutoría para obtener algún resultado más específico y que ellos se sintieran más motivados. Por todo lo expuesto, debemos recordar la importancia de la educación emocional, siendo la encargada de la prevención, al apostar por la fortaleza personal de las generaciones futuras (Pérez \& Filella, 2019).

- Tercera fase: se iniciaría en la segunda mitad del segundo trimestre y se desarrolla a lo largo del resto del curso escolar. Se comienzan a utilizar los mapas conceptuales de forma sistemática por parte de los alumnos y en diferentes materias como evidentemente las asignaturas de idiomas, pero se puede trasladar esta herramienta como es matemáticas para resolver problemas.

- Cuarta fase: tendrá lugar al final del tercer trimestre, está destinada a la evaluación de todo el proceso de intervención y propuestas de mejora de este. Hay que destacar que todas las actividades están recogidas en un diario de clase, donde se anota los aspectos más significativos de cada sesión. Otro aspecto que se ha contado ha sido con una estimación de los docentes con el fin de conocer sus opiniones al respecto. 


\section{Resultados}

En el estudio se verificó que el uso de la herramienta Cmap Tools en el aula virtual mejora el rendimiento general de los alumnos tengan o no tengan dislexia, eso supuso realizar dos medidas pre y postest y comparar si realmente estos alumnos mejoraron en su rendimiento y satisfacción tras la utilización de esta herramienta. En general se pudo observar que después de utilizar la herramienta Cmap Tools en el aula con el alumnado, los resultados respecto al aprendizaje en general y la comprensión lectora en particular, fueron mejores que los resultados obtenidos en el pretest. Para ello se utilizó una escala de identificación de dificultades de aprendizaje (EIDP, enfocada sobre todo en la lectura comprensiva) que fue pasada antes de los talleres y después.

Otro aspecto que se analizó es la autoestima y la motivación por parte del alumnado, comprobando que al igual que en aspecto anterior, los resultados son más favorables tras el empleo de esta herramienta. Para ello se utilizó una escala de satisfacción y bienestar de elaboración propia.

\subsection{Descripción de los resultados encontrados}

Con el fin de identificar los resultados obtenidos por grupo al igual que por la diferenciación de sus participantes, se ha utilizado la siguiente nomenclatura.

Sin Pre Cmap = sin dislexia antes de usar el Cmaps Tools. Con Pre Cmap $=$ con dislexia antes de usar Cmpas Tools.

Sin Post $\mathrm{Cmap}=$ sin dislexia después de usar Cmaps Tools. Con Post $\mathrm{Cmap}=$ con dislexia después de usar Cmpas Tools.

Los porcentajes de los resultados han sido redondeados para no incluir decimales.

En primer lugar, vamos a mencionar los resultados obtenidos por el grupo 1.

Figura 1.

Resultado Grupo 1 en «función de lectoescritura».

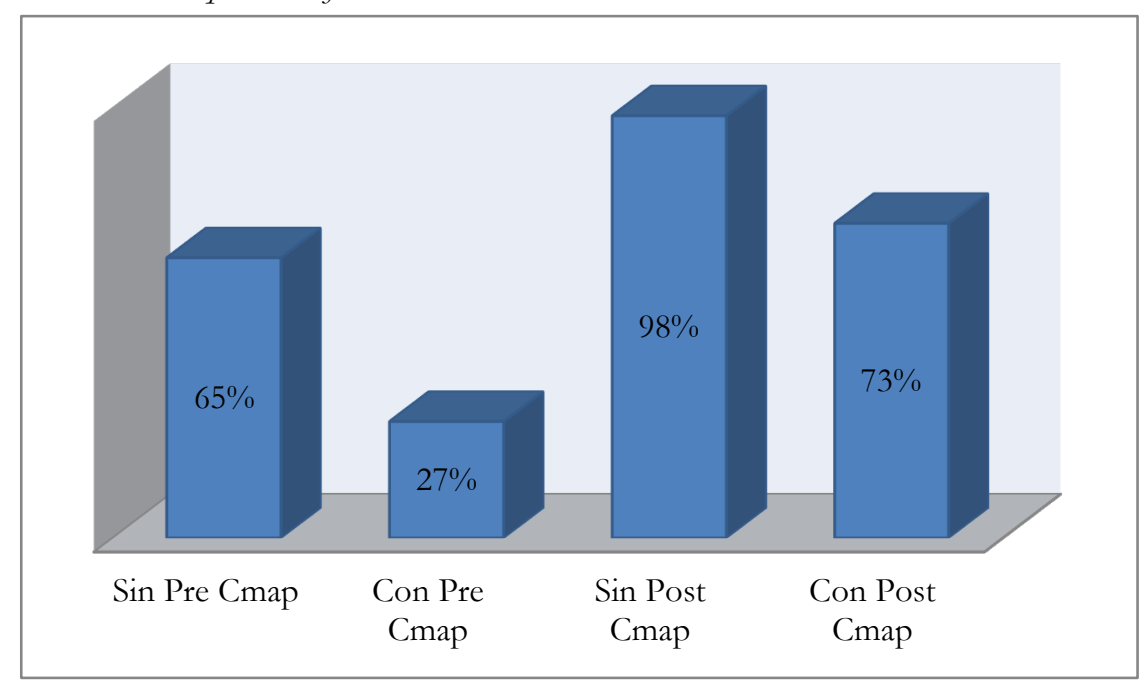

Fuente: elaboración propia.

El primer grafico muestra los resultados a nivel de lectoescritura, que tal y como se observa, los resultados son mejores tras incorporar los Cmaps como herramienta del aprendizaje. Este hecho ocurre tanto para el alumnado con dislexia como para el alumnado que no presenta dificultades de aprendizaje.

En el caso en particular del alumnado con dislexia, los resultados obtenidos mejoran drásticamente y siendo este colectivo el que presenta dificultades, no podemos negar los beneficios que provoca en estos perfiles.

El segundo aspecto analizado es el nivel de motivación tras la utilización de la herramienta Cmaps Tools. 
Figura 2.

Resultado Grupo 1 en «motivación».

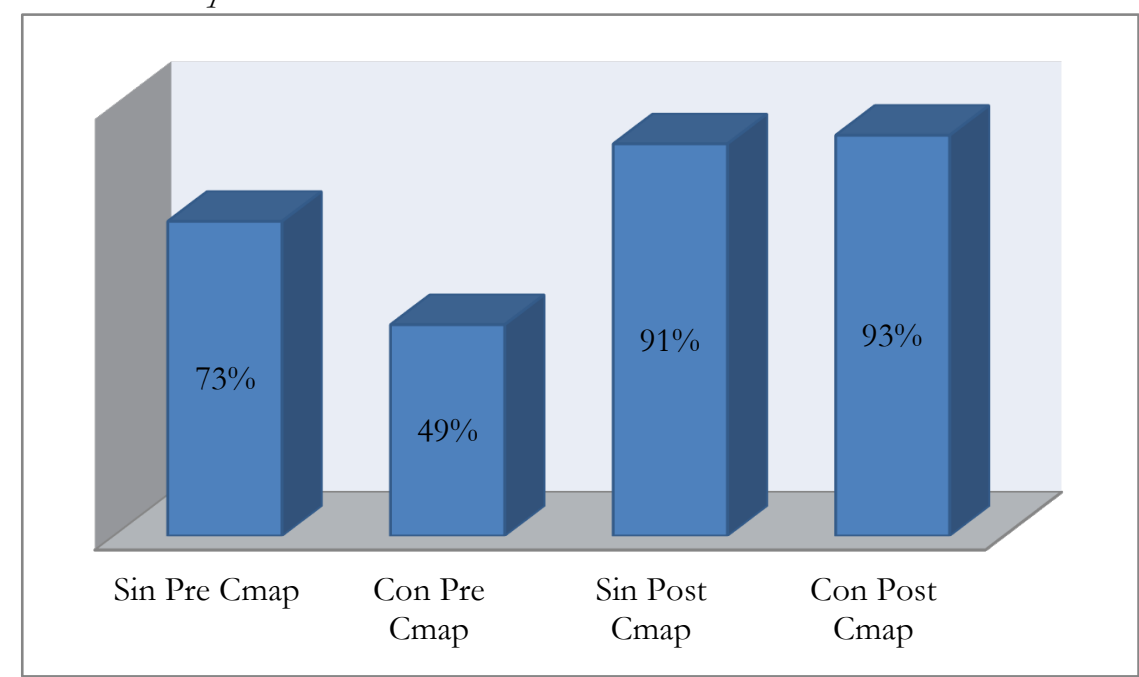

Fuente: elaboración propia.

Los resultados indican que el nivel de motivación en los dos colectivos es mucho más significativo tras emplear la herramienta. Este aspecto nos muestra que el hecho de utilizar otro elemento no tan frecuente, así como diferente y que a su vez incluye aspectos de mejora en el aprendizaje, al alumnado le ha resultado motivador y ha mejorado su satisfacción dentro del grupo.

En los alumnos con dislexia podemos observar como los resultados obtenidos tras utilizar la herramienta han sido mucho más positivos en comparación a los resultados iníciales.

Tras describir los resultados del grupo 1, vamos a analizar los resultados obtenidos por el grupo 2.

Figura 3.

Resultado Grupo 2 en «función de lectoescritura».

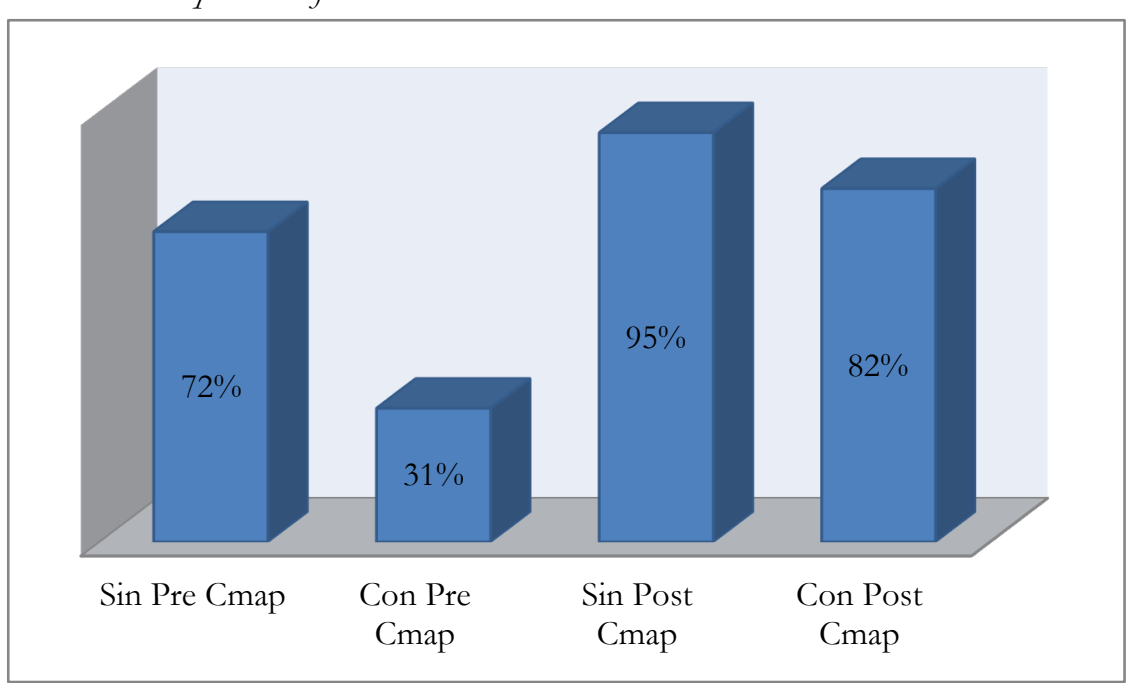

Fuente: elaboración propia.

Este gráfico nos indica que los resultados obtenidos por este grupo a nivel de lectoescritura son mucho mejores tras incorporar la herramienta de Cmaps en los dos colectivos de alumnado, siendo mucho más significativo en el alumnado con dislexia con respecto a los resultados iníciales, no obstante, el grupo que no presenta dificultades de aprendizaje obtiene un mayor resultado global.

El siguiente aspecto por mencionar es el nivel de autoestima que mostraban los alumnos antes y después de trabajar con esta herramienta. 
Figura 4. Resultado Grupo 2 en «autoestima».

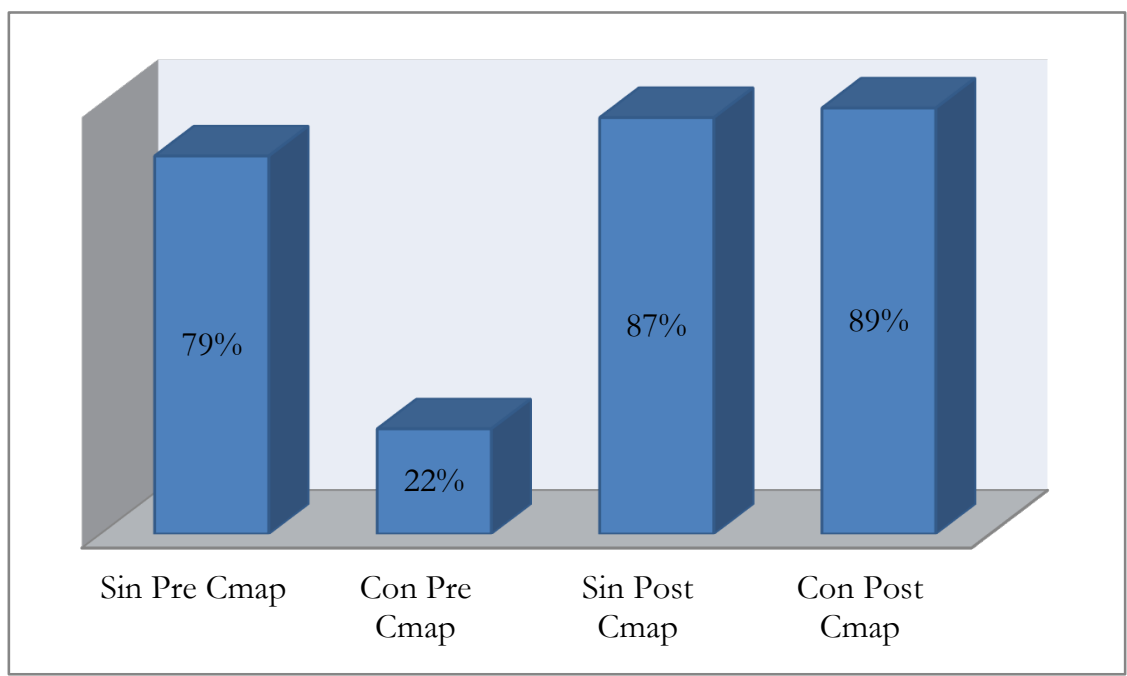

Fuente: elaboración propia.

Si observamos el alumnado que posee dislexia muestra inicialmente unos niveles preocupantes de autoestima, mostrando que este tipo de alumnado necesita unas herramientas de aprendizaje que hagan poder ver resultados en un plazo más corto con el fin de no perder la confianza en ellos mismos. Como dato positivo para el alumnado disléxico, es que, tras la utilización de esta herramienta, realiza un giro muy satisfactorio en este aspecto alcanzando incluso niveles superiores al alumnado sin dislexia, no obstante, en el colectivo sin dificultades de aprendizaje, sigue siendo también positivo para ellos esta herramienta ya que sube positivamente el porcentaje.

El tercer y último aspecto analizado es el nivel de motivación que tiene los alumnos antes y después de la aplicación de la herramienta Cmaps Tools en el aula.

Figura 5. Resultado Grupo 2 en «motivación».

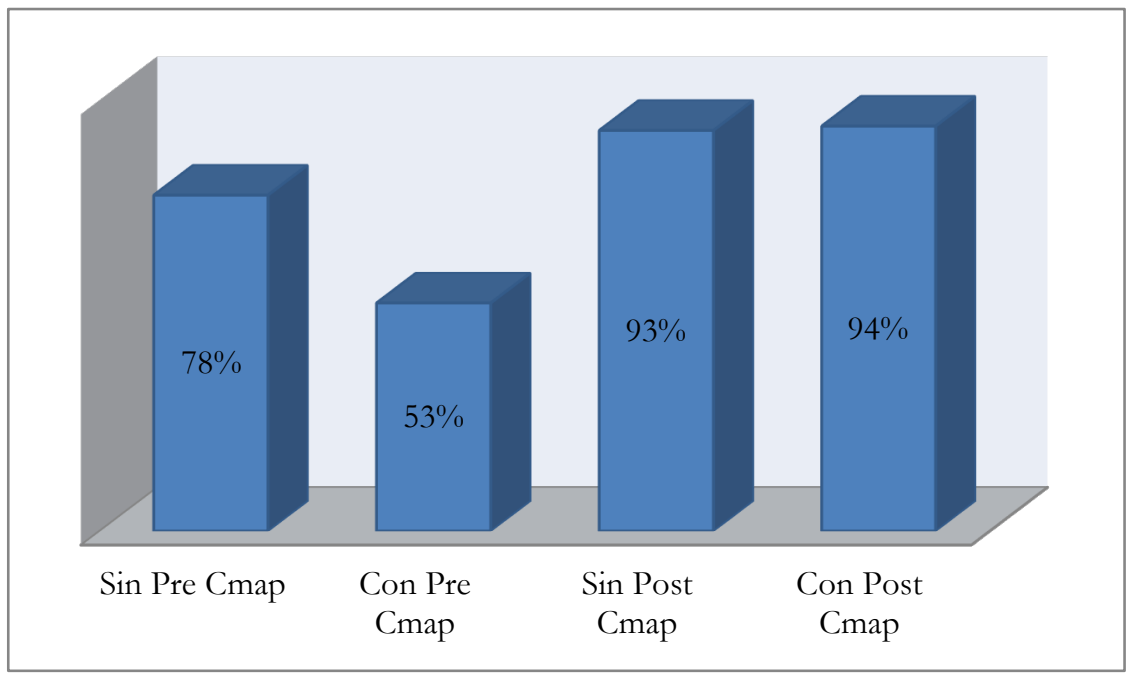

Fuente: elaboración propia.

El gráfico nos muestra que en aspectos generales, los porcentajes son más elevados a nivel global de todo el colectivo, mostrando una media más alta que en las otras cuestiones, por tanto, en cuanto a motivación aunque inicialmente el alumnado con dislexia en este aspecto es menor que el del otro colectivo, tras utilizar la herramienta Cmaps Tools, se ha quedado muy igualado entre los dos colectivos y con porcentajes de motivación muy altos, cuestión muy favorable para incluir esta herramienta en el aula. 


\subsection{Comparativa entre grupos}

A modo comparativo entre grupos, podemos indicar que en grupo 2 se nota una mejora de la comprensión lectora en el alumnado con dislexia frente al grupo 1. En cuanto al alumnado sin dislexia los datos indican que:

En cuanto a la autoestima: es el grupo 1 en su totalidad como tal, inicialmente muestra una autoestima mucho más elevada que el grupo 2, pero tras la aplicación de la herramienta Cmaps Tools, los porcentajes en los dos grupos son muy buenos, demostrando con ello, que en este aspecto mejora consideradamente en el individuo. Como dato específico, los alumnos que no poseen dificultades de aprendizaje del grupo 1 son los que han mostrado un mayor resultado.

Y la última cuestión, la motivación, podemos afirmar que los dos grupos han mejorado notablemente este aspecto, teniendo resultados muy parecidos.

\section{Discusión y Conclusiones}

En definitiva, la atención a la diversidad así concebida se basa en una serie de principios, que sustentan una atención educativa de más calidad, centrada en el diálogo y la toma de decisiones conjuntas. Con el presente trabajo se pretende, crear una línea metodológica innovadora, expandiendo una formación de calidad entre todos los profesionales que atienden al alumnado, y llegar a protocolizar la atención al alumnado con dislexia.

Como perspectiva de futuro, se espera que la metodología de este centro en concreto se pudiera extrapolar al resto de centros de la Comunidad Autónoma a través de formación específica a través de seminarios, grupos de trabajo, en centros de profesorado, o a través de proyectos de innovación educativa (PIE). Como todo proyecto, nos encontramos con una serie de limitaciones que podrían dificultar la puesta en marcha y el transcurso de todas las ideas plasmadas en el presente trabajo. Un ejemplo sería la falta de estabilidad en las plantillas debido a la alta tasa de interinidad que existe en gran parte de los centros educativos. Así mismo, la falta de profesorado de apoyo que junto con la gran ratio de alumnos por aula que encontramos, dificulta la atención individualizada.

Debemos no obstante luchar por mejorar la calidad de la educación y la prestación de ayudas a todo el alumnado. No debemos olvidarnos de ningún tipo de dificultad, y por mucho que sea difícil diagnosticarla, y tener formación para dar solución a cada una de ellas, tenemos que comprometernos con nuestra formación y actualización para dar el mejor de nuestros servicios.

Esta herramienta supone un instrumento de ayuda al tutor a integrar de manera satisfactoria a los alumnos con necesidades de apoyo educativo en áreas tan importante como la compresión lectora.

\section{Referencias}

Bernal, N. C. \& Cerezo, F. (2019). Programa CIE: Intervención en ciberacoso escolar mediante el desarrollo de la Inteligencia Emocional. Creatividad y sociedad: Revista de la Asociación para la Creatividad, (5), 39-49.

Bernal, N.C. \&Cerezo, F. (2019). El programa CIE: Intervención en ciberacoso escolar mediante el desarrollo de la Inteligencia Emocional. European Journal of Health Research, 5(1), 39-49. doi: 10.30552/ejhr.v5i1.136.

Bisquerra, R. (2003). Educación emocional y competencias básicas para la vida. Revista de Investigación Educativa, 21(1), 7-43. Recuperado de https://revistas.um.es/rie/article/view/99071

Cabañas, J. E., \& Ojeda Fernández, Y. M. (2003). Aulas virtuales como herramienta de apoyo en la educación de la Universidad Nacional Mayor de San Marcos. (Tesis de ingeniería). Biblioteca Virtual. Lima, Perú. Recuperado de https://hdl.handle.net/20.500.12672/2534

Cañas, A., \& Ford, K. (2014). Aprendizaje a través de mapas conceptuales, Florida. pp. 195.

Cañas, A. J., Ford, K.M., Hayes, P. J., Reichherzer, T., Suri, N., Coffey, J., et al. (2002). Aprendizaje a través de mapas conceptuales. Institute for Human and Machina Cognition. University of West. Recuperado de http://www.ilhn.com/da- tos/archives/000052.php

Cañas, A. J., Ford, K. M., Hayes, P. J., Reichherzer, T., Suri, N., Coffey, J., et al. (2005). Aprendizaje a través de mapas conceptuales. Institute for Human and Machina Cognition. University of West. Florida. Agosto 2005. 
Cañas, A. J., Ford, K. M., Hayes, P. J., Reichherz, T., Suri, N., Coffey, J., et al. (2005). Aprendizaje a través de mapas conceptuales. Institute for Human and Machina Cognition. Florida: University of West. Recuperado de http://www.ilhn.com/datos/archives/000052.php

Álvarez, C. J. D. (2013). Mapas Mentales y Estilos de Aprendizaje: Aportes a la Enseñanza / Aprendizaje en un Espacio Formativo en Ingenieria. Revista Educación En Ingeniería, 8(16), 45-52. doi: $10.26507 /$ rei.v8n16.360

Fawcett, A., \& Nicolson, R. (2003). El cerebelo: su implicación en la dislexia. Revista Electrónica de Investigación Psicoeducativa y Psicopedagógica, 2(2), 35-58.

González, F. M., \& Iraizoz, N. (2003). El mapa conceptual: un instrumento apropiado para comprender textos expositivos. Navarra: Gobierno de Navarra, Departamento de Educación. Recuperado de https://www.educacion.navarra.es/web/publicaciones/catalogo/-/asset_publis-

her/JONi5m8mCym2/content/el-mapa-conceptual-un-instrumento-apropiado-para-comprender-textos-expositivos

González, B. (2009). Organizadores Gráficos y similares. En, Educación y Pedagogía para el siglo XXI (pp. 13-26). Santiago de Chile.

Lucas-Molina, B., Pérez, A., Fonseca, E., Ortuño, J., Urraca, M., \& Santarén, M. (2017). Fiabilidad y evidencias de validez de un instrumento para la evaluación de la calidad de los mapas conceptuales. Contextos Educativos: Revista de Educación, 2, 119-130. doi: 10.18172/con.3065

Muñoz, J. M., Serrano, R., \& Marín, V. (2014). El aprendizaje colaborativo y su desarrollo a través de mapas mentales. Una innovación educativa en la formación inicial docente. Educatio Siglo XXI, 32, 193-212. doi: $10.6018 / \mathrm{j} / 194151$

Novak, J., \& Gowin, B. (1988). La Didáctica de los Mapas Conceptuales. V. (I). Editorial Emalsa S.A. España. pp. 12- 14.

Pascual, H. (2015). Análisis de la influencia del espacio europeo de educación superior (EEES) en el cambio y la motivación del alumnado universitario y su profesorado en la titulación de magisterio. Estudio comparado con el Reino Unido (Tesis doctoral). Universidad de Granada, Granada, España. Recuperado de https://digibug.ugr.es/handle/10481/42259. URL: http://hdl.handle.net/10481/42259

Pérez, N., \& Filella, G. (2019). Educación emocional para el desarrollo de competencias emocionales en niños y adolescentes. Praxis \& Saber, 10(24), 23-44. doi: 10.19053/22160159.v10.n25.2019.8941

Reina, C., Oliva, A., \& Parra, A. (2010). Percepciones de autoevaluación: Autoestima, autoeficacia y satisfacción vital en la adolescencia. Psychology, Society \& Education, 2(1), 47-59. doi: 10.25115/psye.v2i1.435

Reyes, V., Alcárzar, R. J., Resendiz, A., \& Flores, R. (2017). Miedo, ansiedad y afrontamiento: Estudiantes universitarios mexicanos. Revista Iberoamericana de Psicología, 10(I), 83-92. doi: 10.33881/2027-1786.rip.10110

Segovia, L. (2001). Los Mapas Conceptuales y su aplicación. Lima.

Segovia, L. y Rincón, C. (2011). Los mapas mentales como técnica, mediada por TIC, que contribuye en la disminución de la deserción escolar en Educación Superior: El Caso de la Universidad de La Sabana. Centro de Tecnologías para la Academia Universidad de La Sabana. Recuperado de http://www.virtualeduca.info/ponencias2011/90/Ponencia2.pdf

Scrich, A. J., Cruz, L. A., Bembibre, D., \& Torres, I. (2017). La dislexia, la disgrafia y la discalculia: sus consecuencias en la educación ecuatoriana. Revista Archivo Médico Camagüey, 21(1), 766-772.

Quirant, G. T., Carbonell, N., \& Pascual , H. (2020). El músico y sus diferentes enfermedades profesionales más comunes. Intervención e investigación en contextos clínicos y de la salud, VII, 79- 86. Recuperado de https://ciccs.es/6/contenido/textos/descargar_libro/719.

Quirant, G. T., Pascual, H., \& Carbonell, N. (2020). Trastorno de la ansiedad escénica: Estudio de sintomas comunes en los músicos profesionales. Intervención e investigación en contextos clínicos y de la salud. Volumen (VII) pp.95-102. ASUNIVEP. Recuperado de https://ciccs.es/6/contenido/textos/descargar_libro/789.

Schoeps, K., Tamarit, A., González, R., \& Montoya-Castilla, I. (2018). Competencias emocionales y autoestima en la adolescencia: impacto sobre el ajuste psicológico [Emotional competence and self-esteem in adolescence: Impact on psychological adjustment]. Revista de Psicología Clínica con Niños y Adolescentes, 6(1), 51-56. doi: 10.21134/rpcna.2019.06.1.7

Thompson, M. E. (1992). Dislexia. Su naturaleza, evaluación y tratamiento. Madrid: Alianza.

Ulloa, H., Gutiérrez, M. A., Simancas, I. M., \& Ayala, I. (2018). La motivación a los estudiantes por el profesorado. ¿Se aprende mejor? Revista Educateconciencia, 17(18), 122-130. 
Villalustre, L. \& Del Moral, E. (2010). Mapas conceptuales, mapas mentales y líneas temporales: objetos "de" aprendizaje y "para" el aprendizaje en Ruralnet. Revista Latinoamericana de Tecnología Educativa RELATEC, 9(1), 15-27.

Wolf, M., Vellutino, \& Gleason, J. B. (2000b). Una explicación psicolingüística de la lectura. In J. B. Gleason \& N. Bernstein (Eds.) Psicolinguística (pp. 433-468). Madrid: McGraw-Hill. 